\title{
Putting Evidence to Work
}

A number of global crises usher in the second decade of the new millennium this month: climate change, food security and health among them. Exacerbating each is the world's economic recession that thus far shows little reprieve, especially for poorer nations. In this environment, the logical demand is being raised for science to pay more attention to the public interest and devote more of its efforts to finding solutions to the world's big problems.

The health sector itself has little time and precious few resources to waste. Pressure is mounting to develop an evidence baseborrowing a World Health Organization paradigm — that will get the right products and strategies into the right hands at the right time to do the right thing to improve health for the most people. Such a paradigm requires policymakers to heed science- to listen to medical, population health and social scientists who are committed to advancing health equity by evaluating and proving what works. It also requires fundamental cooperation on all fronts-institutional, local, national and global.

This issue of MEDICC Review is devoted to that particular chain that describes science in the public interest: from research based on public health priorities, to action, and continuous assessment of results to encourage and inform sound policy decisions in a framework of equitable social development.

In the Cuban context, biotechnology R\&D offers one example of an approach that brings research to bear on the lifeand-death issues facing the country's public health system, aiming to develop products for broad application against illnesses accounting for significant disease burden and mortality (Clinical Development and Perspectives of CIMAvax EGF, Cuban Vaccine for Non-small-cell Lung Cancer Therapy). In the process, the some 24 research institutions in the biotech sector have influenced public health strategies. The same principles also foster study to gain deeper understanding of disease as a prerequisite for developing more effective therapies (Hormone Receptors and Other Prognostic Factors in Breast Cancer in Cuba).

Well-directed action and continuous assessment are perhaps nowhere more crucial than in public efforts to confront catastrophe, whether disasters or epidemics. Over the last 30 years, widespread epidemics of meningitis-b, dengue and neuropathy have served to significantly refine Cuban epidemic prevention and control models, generating a legacy that includes a new vaccine, a more robust pediatric intensive care network, and a national early-warning system, the Health Trends Analysis Unit (UATS, its Spanish acronym).

Drawing on such experience and evidence accumulated globally, Cuban government and public health authorities battled the H1N1 flu epidemic in 2009: MEDICC Review brings you the first publication chronicling implementation of this national intersectoral strategy and its preliminary results (Faceoff: Cuba vs H1N1 Influenza).
While incorporation of strong evidence into planning and preparedness for disasters and disease outbreaks is the rule in Cuba, it is less apparent how the results of specific studies or community-based research find their way into national policy discussion. The first step-sharing the evidence-is accomplished by several sets of authors in this issue, one identifying a particular problem (Length of Diagnostic Delay in Patients with Non-small-cell Lung Cancer) and the others potential solutions (Community Involvement in Dengue Vector Control: Cluster Randomised Trial and Effectiveness and Safety of Tisuacryl in Treating Dentin Hypersensitivity).

\section{...advancing health equity by evaluating and proving what works.}

One of the most serious challenges to socially equitable development strategies, and to achieving a healthy population in particular, is the incorporation of intersectoral planning and action. Comparatively little research has been published on the results of such approaches, especially in tackling the social determinants that underly poor health in much of the world. Hopefully, the WHO Commission report on social determinants will prompt more attention to the practice of intersectoral action, and to generating a body of evidence reflecting this experience, as this issue's Viewpoint suggests (Intersectoral Health Strategies: From Discourse to Action).

Also introduced in this issue is Cuba's cochlear implant program, illustrating the delicate balance between access to technology, capacity to apply it, and the potential for enriching quality of life (Breaking the Sound Barrier: Cuba's Cochlear Implant Program). We would encourage the directors of this national effort to publish their scientific and social results.

This MEDICC Review marks our $10^{\text {th }}$ issue as a peer-reviewed publication, and we are pleased to recognize once more the list of collaborators who have made this possible. Our online readership now spans 52 countries, and our Editorial Board is composed of 43 distinguished medical and population health professionals from 14 countries. We take this opportunity to welcome to the Board Dr Linh Cu Le, associate professor at the Hanoi School of Public Health, and Dr Dan Ncayiyana, Director of Benguela Health and Editor-in-Chief of the South African Medical Journal.

Upcoming issues will feature new peer-reviewed sections: Perspectives and Lessons from the Field. In April, our theme will be Child \& Adolescent Health in Cuba. And in July, we look forward to the stewardship of Susan Jupp of the Geneva-based Global Forum for Health Research and Dr Guadalupe Guzmán of Havana's Pedro Kourí Institute of Tropical Medicine, who will guest edit an issue on innovation for health equity. -1 -

\section{The Editors}

